\title{
Possibility of mixed helical p-wave pairings in $\mathrm{Sr}_{2} \mathrm{RuO}_{4}$
}

\author{
Wen Huang $\odot^{1, *}$ and Zhiqiang Wang $\odot^{2, \dagger}$ \\ ${ }^{1}$ Shenzhen Institute for Quantum Science and Engineering \& Guangdong Provincial Key Laboratory of Quantum Science and Engineering, \\ Southern University of Science and Technology, Shenzhen 518055, Guangdong, China \\ ${ }^{2}$ James Franck Institute, University of Chicago, Chicago, Illinois 60637, USA
}

(Received 24 February 2021; revised 11 August 2021; accepted 13 September 2021; published 7 October 2021)

\begin{abstract}
The exact nature of the unconventional superconductivity in $\mathrm{Sr}_{2} \mathrm{RuO}_{4}$ remains a mystery. At the phenomenological level, no superconducting order parameter proposed thus far seems able to coherently account for all essential experimental signatures. Among the latter is the prominent polar Kerr effect, which implies a nonzero ac anomalous Hall conductivity. Assuming the Kerr effect is intrinsic, it can be accounted for by a bulk chiral Cooper pairing with nonzero orbital angular momentum, such as $p+i p$ or $d+i d$, which, however, has difficulties in being reconciled with other experimental results. Given the situation, in this paper we propose alternative possibilities with complex mixtures of distinct helical p-wave order parameters, namely $A_{1 u}+i A_{2 u}$ and $B_{1 u}+i B_{2 u}$ in group theory nomenclature. These states essentially consist of two copies of chiral p-wave pairings with opposite chirality and different pairing amplitudes, and therefore they support intrinsic Hall and Kerr effects. We further show that these states exhibit salient features that may explain several other key observations in this material, including the absence of spontaneous edge current, a substantial Knight shift drop, and possibly signatures in uniaxial strain and ultrasound measurements.
\end{abstract}

DOI: 10.1103/PhysRevResearch.3.L042002

The nature of unconventional superconductivity in $\mathrm{Sr}_{2} \mathrm{RuO}_{4}$ is a puzzle that remains unsolved [1-10]. Its normal state properties have been characterized with unprecedented accuracy - an incredible feat that is not often seen in other unconventional superconductors. The electron correlation in this material is moderate, and superconductivity emerges out of a well-behaved Fermi liquid [3]. These have led to the popular perception that identifying its pairing symmetry and resolving its pairing mechanism should be well within the reach of established theories and experimental techniques. Yet, despite tremendous efforts over the past 20-plus years, neither of these two key issues has been settled. This is largely due to the lack of an order parameter that could coherently interpret all the essential experimental observations $[9,10]$.

Arguably, one principal property of the superconductivity in $\mathrm{Sr}_{2} \mathrm{RuO}_{4}$ is the condensation of at least two superconducting order parameters. This has been inferred from a variety of experiments, including $\mu$ SR [11,12], optical polar Kerr effect [13], Josephson interferometry [14], ultrasound [15,16], etc. A multicomponent pairing is realized if the Cooper pairing is developed in a multidimensional irreducible representation (irrep) of the crystal point symmetry group. One notable example is the chiral $\mathrm{p}$-wave pairing, i.e., $\left(k_{x}+i k_{y}\right) \hat{z}$, which

\footnotetext{
*huangw3@sustech.edu.cn

†zqwang@uchicago.edu
}

Published by the American Physical Society under the terms of the Creative Commons Attribution 4.0 International license. Further distribution of this work must maintain attribution to the author(s) and the published article's title, journal citation, and DOI. belongs to the $E_{u}$ irrep of the $D_{4 h}$ group. Here, $\hat{z}$ denotes the orientation of the so-called $\mathbf{d}$-vector, which describes the spin configuration of a spin-triplet pairing [3]. Another possibility is coexisting order parameters from distinct one-dimensional irreps. In this scenario, the multiple components typically condense below different critical temperatures except for accidental degeneracy.

In light of the evidence of time-reversal symmetry breaking from the $\mu \mathrm{SR}$ and the polar Kerr effect measurements [11-13], the multiple components presumably form a complex pairing, such that the state does not return to itself after a time-reversal operation. The Kerr effect, as well as the closely related anomalous Hall effect, intuitively implies an underlying pairing with finite orbital angular momentum (such as $p+i p, d+i d$, etc.), as has been substantiated by a recent study [17]. Hence, if the Kerr effect in $\mathrm{Sr}_{2} \mathrm{RuO}_{4}$ is intrinsic (i.e., not related to extrinsic effects such as impurities or lattice dislocations), its superconducting symmetry is limited to a few candidates. However, as it currently stands, none of the chiral superconducting states appears able to reconcile all of the key observations [17]. For example, although $p_{x}+i p_{y}$ or $d_{x z}+i d_{y z}$ pairing can naturally explain the Kerr effect, the former is strongly disfavored by the recent NMR measurements[18,19], and both generally exhibit finite spontaneous surface current that is not detected [20,21].

In this work, we turn to the helical p-wave pairings $\left(A_{1 u}\right.$, $A_{2 u}, B_{1 u}$, and $B_{2 u}$ irreps), which are analogs of the ${ }^{3} \mathrm{He}$ $\mathrm{B}$-phase [22]. The four pairings individually preserve timereversal symmetry, as each effectively consists of a pair of sub-Hilbert space characterized, respectively, by $k_{x}+i k_{y}$ and $k_{x}-i k_{y}$ pairings with identical gap amplitude. However, an appropriate complex mixture of them could tune the gap 


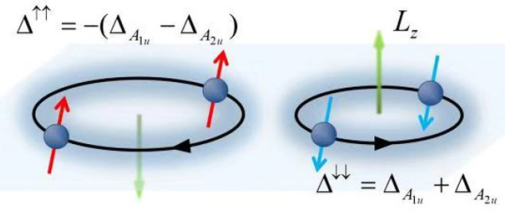

FIG. 1. Illustration of the Cooper pairing in the mixed helical pwave $A_{1 u}+i A_{2 u}$ state in a single-orbital model. The state consists of two copies of the chiral p-wave model with opposite chirality. The Cooper pairs are drawn with different sizes to reflect their different pairing amplitudes. Green arrows indicate the direction of the Cooper pair orbital angular momentum $L_{z}$. Another candidate state $B_{1 u}+$ $i B_{2 u}$ has a similar pairing configuration.

amplitude ratio between $k_{x}+i k_{y}$ and $k_{x}-i k_{y}$ away from unity (see Fig. 1); the resultant "asymmetric" state then has a net chirality and supports an intrinsic Kerr effect. Among all possible two-component order-parameter combinations of the four helical pairings, we identify two that meet our requirement: $A_{1 u}+i A_{2 u}$ and $B_{1 u}+i B_{2 u}$.

The idea is most transparent when presented in a singleorbital model. Take $A_{1 u}+i A_{2 u}$ as an example: in terms of the d-vector, the pairing functions of $A_{1 u}$ and $A_{2 u}$ are given, respectively, by $k_{x} \hat{x}+k_{y} \hat{y}$ and $k_{x} \hat{y}-k_{y} \hat{x}$. The full gap function then becomes

$$
\mathbf{d}_{\mathbf{k}}=\Delta_{A_{1 u}}\left(k_{x} \hat{x}+k_{y} \hat{y}\right)+i \Delta_{A_{2 u}}\left(k_{x} \hat{y}-k_{y} \hat{x}\right),
$$

where $\Delta_{A_{1(2) u}}$ are real gap amplitudes. $\mathbf{d}_{\mathbf{k}}$ is nonunitary since $\mathbf{d}_{\mathbf{k}}^{*} \times \mathbf{d}_{\mathbf{k}} \neq 0$ [22], implying unequal pairing gap amplitudes for the two spin species: $\Delta^{\uparrow \uparrow}(\mathbf{k})=-\left(\Delta_{A_{1 u}}-\Delta_{A_{2 u}}\right)\left(k_{x}-i k_{y}\right)$ for spin-up and $\Delta^{\downarrow \downarrow}(\mathbf{k})=\left(\Delta_{A_{1 u}}+\Delta_{A_{2 u}}\right)\left(k_{x}+i k_{y}\right)$ for spindown (Fig. 1). If $\Delta_{A_{1 u}}=\Delta_{A_{2 u}}$, the state resembles the ${ }^{3} \mathrm{He} A_{1^{-}}$ phase, which has one spin component unpaired [22]. More generally, both spin species are paired and they carry opposite orbital chiralities, i.e., opposite $L_{z}$. Such a state is odd-parity by nature [23], and it exhibits other salient features potentially consistent with the absence of spontaneous current signature [20,21] and the substantial Knight shift drop [18,19], as we shall see later. Since the $B_{1 u}+i B_{2 u}$ state essentially shares the same general features, we will not present further analyses about it in the remainder of the paper.

At this point, it is worth pointing out that multiple microscopic calculations [24-30] have found that helical pairing states are favored in certain reasonable interaction parameter space. More interestingly, in some cases the splitting among various helical states could be rather small [30], making it reasonable to consider accidentally degenerate pairing. Here, we make no attempt to delve into the pairing mechanism or how the pairing is distributed among different orbitals in $\mathrm{Sr}_{2} \mathrm{RuO}_{4}$. Instead, we will focus on the general phenomena that do not rely on microscopic details. Apart from some occasional general discussions, our analyses will be largely based on a concrete two-orbital model containing the $\mathrm{Ru} d_{x z}$ and $d_{y z}$ orbitals. As far as the physical properties to be discussed are concerned, we expect the same qualitative features for more general multiorbital models of $\mathrm{Sr}_{2} \mathrm{RuO}_{4}$. For simplicity, we assume the simple pairing functions for the various odd-parity (p-wave) superconducting channels as given in Table I.

\section{I. ac ANOMALOUS HALL EFFECT AND KERR ROTATION}

Since our proposed state is essentially composed of a pair of (spinless) chiral p-wave models with opposite chirality and inequivalent gap amplitudes, we expect it to support the anomalous Hall effect. Notably, while the intrinsic anomalous Hall effect is generally absent in single-orbital chiral superconducting models [31-34], $\mathrm{Sr}_{2} \mathrm{RuO}_{4}$ has an inherent multiorbital character.

Below we demonstrate the intrinsic ac anomalous Hall effect and Kerr rotation for the $A_{1 u}+i A_{2 u}$ state, using the same two-orbital model as in Ref. [32]. Without qualitative impacts on our results, we neglect spin-orbital coupling (SOC) in this section for simplicity. Then the spin-up and -down blocks decouple, and their contributions to the two-dimensional Hall conductivity $\sigma_{\mathrm{H}}$ can be computed separately. Here, we briefly describe the spin-up block of the model. Written in the basis $\left(c_{d_{x z}, \uparrow}(\mathbf{k}), c_{d_{y z}, \uparrow}(\mathbf{k})\right)$, where $c_{d_{x z}, \uparrow}$ and $c_{d_{y z}, \uparrow}$ are annihilation operators for the $d_{x z}$ and $d_{y z}$ orbitals of $\mathrm{Sr}_{2} \mathrm{RuO}_{4}$, the normal state Hamiltonian reads

$$
H_{\mathrm{N}}(\mathbf{k})=\left(\begin{array}{cc}
\xi_{x z}(\mathbf{k}) & \lambda(\mathbf{k}) \\
\lambda(\mathbf{k}) & \xi_{y z}(\mathbf{k})
\end{array}\right)
$$

where $\quad \xi_{x z}(\mathbf{k})=-2 t \cos k_{x}-2 \tilde{t} \cos \left(k_{y}\right)-\mu, \quad \xi_{y z}(\mathbf{k})=$ $-2 \tilde{t} \cos k_{x}-2 t \cos \left(k_{y}\right)-\mu$, and $\lambda(\mathbf{k})=4 t^{\prime} \sin k_{x} \sin k_{y}$. Here $t$ labels the nearest-neighbor hopping integral of the $d_{x z(y z)}$ orbital along the $x(y)$ direction, $\tilde{t}$ is that of the $d_{x z(y z)}$ orbital along the $y(x)$ direction, and $t^{\prime}$ denotes the hybridization of the two orbitals between next-nearest-neighboring sites. The corresponding BdG Hamiltonian follows as

$$
H_{\mathrm{BdG}}^{\uparrow}(\mathbf{k})=\left(\begin{array}{cc}
H_{\mathrm{N}}(\mathbf{k}) & \hat{\Delta}^{\uparrow \uparrow}(\mathbf{k}) \\
{\left[\hat{\Delta}^{\uparrow \uparrow}\right]^{\dagger}(\mathbf{k})} & -H_{\mathrm{N}}^{T}(-\mathbf{k})
\end{array}\right),
$$

where, following Table I,

$$
\hat{\Delta}^{\uparrow \uparrow}(\mathbf{k})=-\left(\Delta_{A_{1 u}}-\Delta_{A_{2 u}}\right)\left(\begin{array}{cc}
\sin k_{x} & 0 \\
0 & -i \sin k_{y}
\end{array}\right) .
$$

TABLE I. Representative simple odd-parity pairing gap functions (d-vectors) for the two-orbital model containing the Ru $d_{x z}$ and $d_{y z}$ orbitals.

\begin{tabular}{lcr}
\hline \hline irrep & $\mathbf{d}_{x z}$ & $\mathbf{d}_{y z}$ \\
\hline$A_{1 u}$ & $\sin k_{x} \hat{x}$ & $\sin k_{y} \hat{y}$ \\
$A_{2 u}$ & $\sin k_{x} \hat{y}$ & $-\sin k_{y} \hat{x}$ \\
$B_{1 u}$ & $\sin k_{x} \hat{x}$ & $-\sin k_{y} \hat{y}$ \\
$B_{2 u}$ & $\sin k_{x} \hat{y}$ & $\sin k_{y} \hat{x}$ \\
$E_{u}$ & $\sin k_{x} \hat{z}$ & $\sin k_{y} \hat{z}$ \\
\hline \hline
\end{tabular}




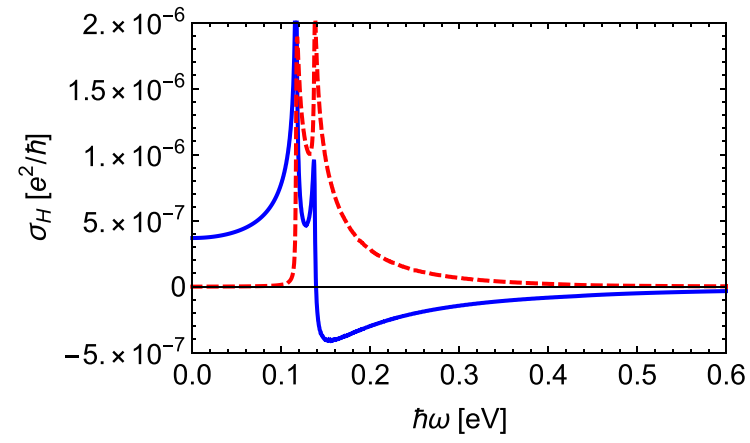

FIG. 2. Real (blue solid line) and imaginary (red dashed line) parts of the $\sigma_{\mathrm{H}}(\omega)$ as a function of $\omega$ for $T=0$. Band parameters are [32] $t=\mu=0.4 \mathrm{eV}, t^{\prime}=0.05 t$ and $\tilde{t}=0.1 t . \Delta_{A_{1 u}}=2 \Delta_{A_{2 u}}=$ $\frac{2}{3} \Delta_{\max }$, with $\Delta_{\max }=\left|\Delta_{A_{1 u}}\right|+\left|\Delta_{A_{2 u}}\right|=0.23 \mathrm{meV}$ [32]. We choose $\left|\Delta_{A_{1 u}}\right| \neq\left|\Delta_{A_{2 u}}\right|$ so that the gap magnitudes are nonzero for both spin up and down; otherwise, one spin component would remain normal at zero temperature, which is not observed.

Using $H_{\mathrm{BdG}}^{\uparrow}$, we calculate $\sigma_{\mathrm{H}}^{\uparrow}(\omega)$ from the standard Kubo formula (without vertex corrections). Details of the calculation can be found in the Supplemental Material [35]. The same calculation is done for $\sigma_{\mathrm{H}}^{\downarrow}$, and the numerical result of the total $\sigma_{\mathrm{H}}$ for temperature $T=0$ is presented in Fig. 2. Not surprisingly, $\sigma_{\mathrm{H}}$ is nonzero; both its real and imaginary parts have a similar frequency dependence to those obtained for a chiral p-wave pairing [32]. The overall magnitude of $\sigma_{\mathrm{H}}$ is reduced by a factor of 2 compared to that in Ref. [32], because in the current scenario $\sigma_{\mathrm{H}}^{\uparrow}$ and $\sigma_{\mathrm{H}}^{\downarrow}$ partially cancel each other due to the opposite chirality of $\hat{\Delta}^{\uparrow \uparrow}$ and $\hat{\Delta}^{\downarrow \downarrow}$, which dictates the signs of $\sigma_{\mathrm{H}}^{\uparrow}$ and $\sigma_{\mathrm{H}}^{\downarrow}$, respectively. On the other hand, such a cancellation is absent in the chiral p-wave pairing case.

We note that $\operatorname{Im}\left[\sigma_{\mathrm{H}}(\omega)\right]$ becomes nonzero at $\omega \gtrsim 0.4 \mathrm{eV}$ for the chosen band parameters. This frequency is determined by the minimum energy cost to create a pair of Bogoliubov quasiparticles from different bands, which is not determined by $2\left|\Delta^{\uparrow \uparrow}\right|$ or $2\left|\Delta^{\downarrow \downarrow}\right|$ but by the hopping parameter $t^{\prime}$ [32]. Therefore, even though $\left|\Delta^{\uparrow \uparrow}\right|$ and $\left|\Delta^{\downarrow \downarrow}\right|$ can be quite different, the onset frequencies of $\operatorname{Im}\left[\sigma_{\mathrm{H}}^{\uparrow}\right]$ and $\operatorname{Im}\left[\sigma_{\mathrm{H}}^{\downarrow}\right]$ are actually almost identical.

From the calculated $\sigma_{\mathrm{H}}$, one can estimate the Kerr rotation angle using

$$
\theta_{\mathrm{K}}(\omega)=\frac{4 \pi}{\omega d} \operatorname{Im}\left[\frac{\sigma_{\mathrm{H}}(\omega)}{n\left(n^{2}-1\right)}\right]
$$

where $n=n(\omega)$ is the complex index of refraction, and $d$ is the interlayer spacing of $\mathrm{Sr}_{2} \mathrm{RuO}_{4}$ along the $c$-axis. $n(\omega)$ can be estimated from the longitudinal optical conductivity, which is modeled by a Drude model as in Ref. [32]. For details, see Ref. [35]. The estimated $\theta_{\mathrm{K}}(\hbar \omega=0.8 \mathrm{eV}) \approx 20 \mathrm{nrad}$ [35], which may potentially account for the experimental value of 65 nrad observed by Xia et al. [13], given the uncertainty in our estimate of $n(\omega)$ as well as the neglect of the $\gamma$ band in the current calculation. We note that $\sigma_{\mathrm{H}}$, and therefore the estimated Kerr angle $\theta_{\mathrm{K}}$, does depend on the actual ratio between $\left|\Delta_{A_{1 u}}\right|$ and $\left|\Delta_{A_{2 u}}\right|$. However, the dependence is quite weak as long as the two are comparable [35].

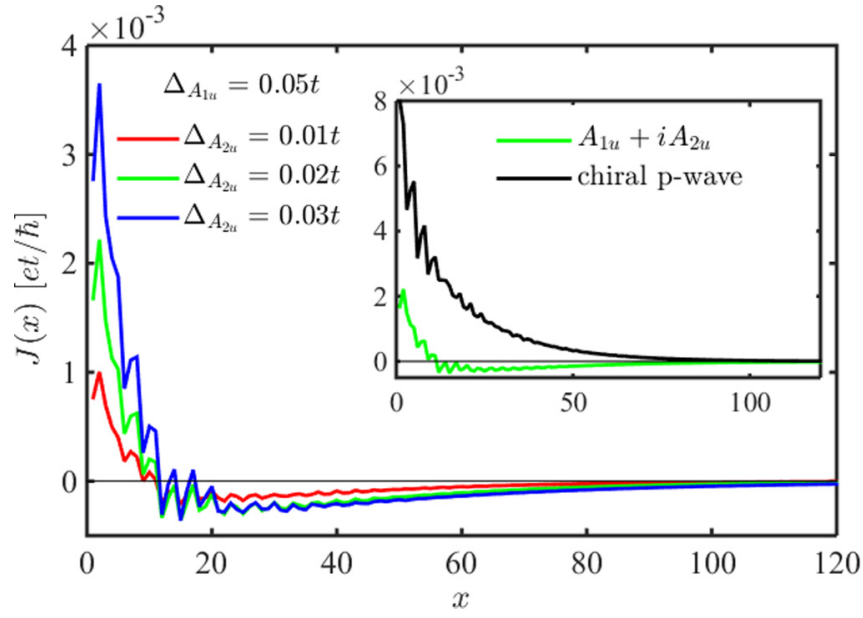

FIG. 3. Distribution of zero-temperature spontaneous edge current in our two-orbital model with a mixed helical p-wave $A_{1 u}+i A_{2 u}$ pairing. The $A_{1 u}$ component is held fixed at $\Delta_{A_{1 u}}=0.05 t$. Inset: a comparison of the spontaneous current of the mixed helical and chiral p-wave pairing states with similar gap amplitudes. These calculations follow those in Refs. [39-41], and the lattice constant is defined to be unity.

\section{SPONTANEOUS CURRENT}

A chiral p-wave state shall generate finite spontaneous surface current [36,37]. Following the original proposal of chiral p-wave pairing in $\mathrm{Sr}_{2} \mathrm{RuO}_{4}$, attempts to detect signatures of such a current have had little success, suggesting that the spontaneous current is either simply absent or too tiny to be resolved in actual measurements [20,21].

The $A_{1 u}+i A_{2 u}$ state has just the right appeal for explaining this, which is already obvious in the single-orbital case. Here, the two spin species each generates an edge current, and these currents flow in opposite direction due to the opposite chirality. Moreover, the integrated current in fact vanishes in the BCS limit. However, thanks to the distinct decay length scales of the two contributions, the current distribution does not exactly cancel at each spatial point.

Going beyond single-orbital model, the integrated current may not vanish due to SOC and other band structure effects. However, the strong suppression persists, as is demonstrated in Fig. 3 for our two-orbital model. Neglecting the fast Friedel oscillations, the spontaneous current generally contains two canceling contributions characterized by different decay length scales. For the calculations shown in the figure, the net current in the mixed helical state is typically more than 20 times smaller than that in a chiral p-wave state with similar gap magnitudes (see the inset of Fig. 3).

It should be stressed that such suppression is inherent, as it is achieved without appealing to surface disorder, anisotropic band and gap structure - factors typically having the potential to further reduce the spontaneous current, as is known for the case of chiral p-wave [38-44]. In fact, this behavior is also expected from the Ginzburg-Landau theory, at the lowest order of which the spontaneous current is related to crossgradient bilinears of the involving order parameters, such as $\partial_{x} \Delta_{A_{1 u}}^{*} \partial_{y} \Delta_{A_{2 u}}[43,45]$. In the present case, this lowest order contribution vanishes by symmetry [35], and higher order 


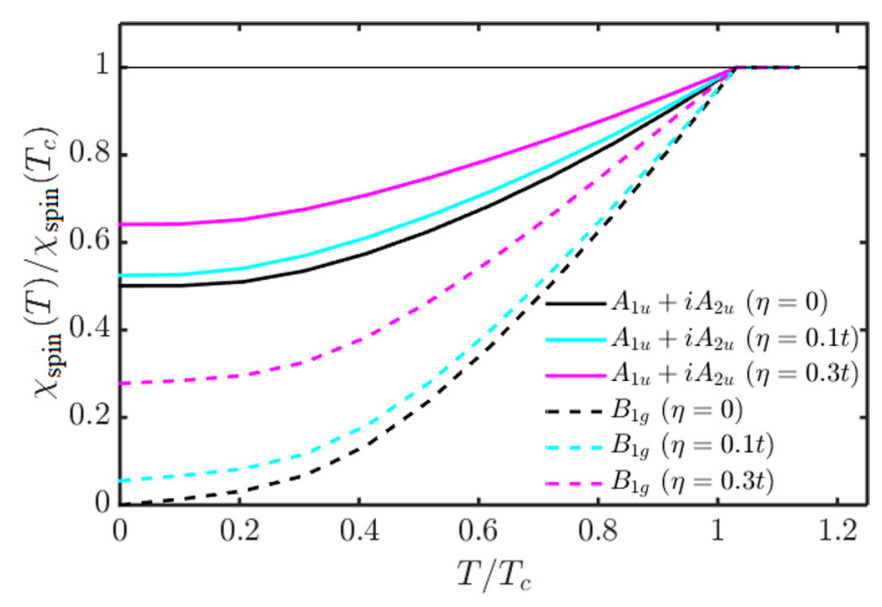

FIG. 4. Temperature dependence of the spin susceptibility in the $A_{1 u}+i A_{2 u}$ (solid curves) and $B_{1 g}$ (dashed curves) superconducting states, in the presence of a small Zeeman field in the $x$-direction. Here, $\eta$ denotes the strength of SOC.

terms such as $\partial_{x}^{3} \Delta_{A_{1 u}}^{*} \partial_{y} \Delta_{A_{2 u}}$ are needed to account for the small local current distribution. By the same token, similar physics applies to the chiral $d_{x^{2}-y^{2}}+i d_{x y}$ pairing [46]. In passing, we note that for the $d_{x^{2}-y^{2}}+i g_{x y\left(x^{2}-y^{2}\right)}$ pairing [47] the spontaneous current is in general finite at the (100) surfaces and vanishes at the (110) surface, analogous to the situation in $s+i d_{x y}$ pairing [37].

Finally, the suppression in the $A_{1 u}+i A_{2 u}$ phase is also independent of the edge orientation, and the same phenomenology takes place at the domain walls separating regions of time-reversed pairings.

\section{SPIN SUSCEPTIBILITY AND KNIGHT SHIFT}

Recent revised NMR measurements under external inplane magnetic field reveal a substantial Knight shift drop below $T_{c}[18,19]$. This implies a drop of spin susceptibility, $\chi_{\text {spin }}$, which was subsequently confirmed by a polarized neutron scattering study [48]. A later NMR study further places an upper bound of $10 \%$ of the normal state susceptibility at the lowest measured temperatures [49]. These reports can be most straightforwardly explained in terms of spin-singlet pairing. However, as the observed Knight shift contains contributions from both spin and orbital degrees of freedom, the exact fraction of the spin susceptibility drop in the zero field limit may deserve a more careful inquiry [10]. As we argue below, our proposed states may still have the potential to reconcile with the susceptibility drop.

Owing to the in-plane d-vector orientation, the mixed helical states naturally exhibit a reduced $\chi_{\text {spin }}$ across $T_{c}$ under the in-plane field. In fact, in the absence of SOC, $\chi_{\text {spin }}$ at $T=0$ equals half of its normal state value. This is demonstrated in Fig. 4, which shows the temperature-dependent susceptibility in the presence of a weak Zeeman field directed along the $x$-direction. For comparison, we also show results for a spin-singlet d-wave state (a pairing in the $B_{1 g}$ irrep). In the calculations, we have assumed that the two helical pairings onset at the same critical temperature, and that their temperature dependence follows the standard BCS mean-field behavior.
The SOC may facilitate extra spin-flipping processes that could have otherwise been suppressed by Cooper pairing, thereby alleviating the drop of $\chi_{\text {spin }}$. This is exemplified by the noticeable increase of $\chi_{\text {spin }}(T=0)$ with enhanced SOC (Fig. 4). Notably, even a pure spin-singlet pairing may develop a residual $\chi_{\text {spin }}(T=0)$ [27,29]. For the two-orbital model in question, this can be explained in the following terms. Here, SOC takes the form of $\eta \hat{L}_{z} \hat{s}_{z}$ where $\hat{L}_{z}$ operates on the orbital degrees of freedom and $\hat{s}_{z}$ is the spin Pauli matrix. To be specific, $\hat{L}_{z}=-i \epsilon_{i j}$ [30] with $\epsilon_{i j}$ the fully antisymmetric tensor and $\{i, j\}=\{1,2\}$ representing the $\left\{d_{x z}, d_{y z}\right\}$ orbitals in Eq. (2). The SOC term flips the in-plane spin components but not the ones along $z$. As a consequence, upon turning on a finite $\eta$, the $z$-component of the spin susceptibility $\chi_{\text {spin }}^{z z}$ remains fully suppressed at $T=0$ (not shown in Fig. 4), whereas the $x$ component, $\chi_{\text {spin }}^{x x}$, acquires a residual value. We expect such an enhancement of $\chi_{\text {spin }}(T=0)$ due to SOC to persist in a more general multi-orbital model of $\mathrm{Sr}_{2} \mathrm{RuO}_{4}$ [50-52]. Finally, it is worth remarking that, the natural mixing of (real)-spin triplet and singlet pairings in the presence of SOC [50,53], which is not considered here, may also introduce corrections to the spin susceptibility.

Note that our calculations were done in the noninteracting limit. Correlations renormalize the normal and superconducting state $\chi_{\text {spin }}$ in different manners, reducing the ratio $\chi_{\text {spin }}(T=0) / \chi_{\text {spin }}\left(T=T_{c}\right)$ from its non-interacting value [54]. Based on an estimate of the Wilson ratio of $2[19,55]$ and using the data for $\eta=0.1 t$ in Fig. 4 , we obtain $\chi_{\text {spin }}(T=$ $0) / \chi_{\text {spin }}\left(T=T_{c}\right) \approx 35 \%$ for the $A_{1 u}+i A_{2 u}$ state. This is a substantial overall suppression, although, at face value, still noticeably higher than the upper bound of $10 \%$ suggested by the most recent NMR measurement [49]. However, our crude approximation could be insufficient for a quantitative comparison, and a calculation based on a more realistic correlated multiorbital model may be needed. Furthermore, the potential complications in disentangling the orbital and spin contributions to the Knight shift $[10,56]$ could affect the experimental interpretations.

\section{STRAIN AND ULTRASOUND}

Uniaxial strain and ultrasound measurements reveal crucial information about the symmetry of the superconducting order $[15,16,57]$. Most informative among the existing experiments are the ones that probe the coupling between the order parameters and the $B_{1 g}\left(\epsilon_{x^{2}-y^{2}}\right)$ and $B_{2 g}\left(\epsilon_{x y}\right)$ strains. For our mixed helical state, the leading-order coupling has the following form in the Ginzburg-Landau free energy:

$$
f_{\text {coupling }}=\sum_{i} \epsilon_{i}^{2}\left(\alpha_{i}\left|\Delta_{A_{1 u}}\right|^{2}+\beta_{i}\left|\Delta_{A_{2 u}}\right|^{2}\right),
$$

where $i=x^{2}-y^{2}, x y$ for the $B_{1 g}$ and $B_{2 g}$ strains, respectively, and $\alpha_{i}\left(\beta_{i}\right)$ are coupling constants. No other order parameter bilinears are present. This free energy has two implications. On the one hand, the mean-field critical temperatures of the two components shall both follow a quadratic dependence on the strain. This seems to agree with the observed behavior in several uniaxial strain measurements $[58,59]$. We do note that there is no solid evidence of the expected split of transitions in thermodynamic measurements $[60,61]$, although this could 
be explained away by arguments unrelated to symmetry [62]. On the other hand, the absence of a linear coupling to $\epsilon_{i}$ in Eq. (6) disallows any discontinuity in the shear elastic moduli $\left(c_{11}-c_{12}\right) / 2$ or $c_{66}$. This stands in contrast with recent reports, where a jump in the latter has been observed $[15,16]$. Interestingly, a linear coupling to the $B_{2 g}$ strain (associated with $c_{66}$ ) is possible for mixed $A_{1 u}$ and $B_{2 u}$, as well as mixed $A_{2 u}$ and $B_{1 u}$ states [30]. This suggests a way to reconcile our proposed order with the ultrasound experiments: the breaking of crystalline rotational symmetry around lattice dislocations can allow the $B_{1 u}$ and/or $B_{2 u}$ order parameters to locally condense against the backdrop of a bulk $A_{1 u}+i A_{2 u}$ order, leading to a local mixture of $A_{1 u}$ with $B_{2 u}$ (and/or $A_{2 u}$ with $B_{1 u}$ ) that couples linearly to the $B_{2 g}$ strain.

\section{CONCLUDING REMARKS}

Under the assumption that the observed polar Kerr effect is a genuine response of superconducting $\mathrm{Sr}_{2} \mathrm{RuO}_{4}$ in the clean limit, we proposed that the mixed helical p-wave states, $A_{1 u}+i A_{2 u}$ and $B_{1 u}+i B_{2 u}$, represent faithful alternative candidates to chiral superconducting states. We also discussed how these states may be compatible with several other key measurements. Interestingly, a recent study [63] shows that a helical state may also explain the Pauli limiting behavior of the in-plane $H_{c 2}$ and the first-order phase transition observed in the $H-T$ phase diagram at low $T$ and at $H$ near $H_{c 2}[64,65]$. Nevertheless, a full reconciliation with other important observations may require further examination. For example, the presence of gapless excitations [66-70] would place a strong constraint on the detailed forms of the $\mathrm{p}$-wave pairings. To this end, we take note of the multitude of possibilities made available by the multiorbital nature of this material [50-52,71-79].
Due to their nonunitary nature, our proposed states are also expected to exhibit finite spin polarization in the bulk, which scales as $\left(\Delta / E_{F}\right)^{2}$. The resultant spontaneous bulk magnetization may be probed in SQUID measurements. One such attempt was said to be impeded by the Meissner effect [80], while the existing scanning SQUID [20,21] techniques may need improved precision to resolve the resultant weak magnetization on the sample surface.

As a final remark, the $B_{1 g}+i B_{2 g}$ state (chiral $d_{x^{2}-y^{2}}+i d_{x y}$ pairing) also carries the essential features needed to explain the various observations discussed in this work. Alternatively, in a spirit similar to that proposed in Ref. [81], one can consider that one component of the $B_{1 g}+i B_{2 g}$ state is favored in the clean limit, while the other component condenses around lattice dislocations.

Note added. After posting our manuscript on arXiv, we were informed of another work [82], which discusses the spontaneous magnetization of the $A_{1}+i A_{2}$ and $B_{1}+i B_{2}$ superconducting states in a noncentrosymmetric system with $C_{4 v}$ point group symmetry.

\section{ACKNOWLEDGMENTS}

We acknowledge helpful discussions with Weiqiang Chen, Catherine Kallin, Yongkang Luo, Aline Ramires, Thomas Scaffidi, and Fuchun Zhang. W.H. is supported by NSFC under Grant No. 11904155, the Guangdong Provincial Key Laboratory under Grant No. 2019B121203002, and a Shenzhen Science and Technology Program (KQTD20200820113010023). Z.W. acknowledges financial support from the James Franck Institute at the University of Chicago. Computing resources are provided by the Center for Computational Science and Engineering at Southern University of Science and Technology.
[1] Y. Maeno, H. Hashimoto, K. Yoshida, S. Nishizaki, T. Fujita, J. G. Bednorz, and F. Lichtenberg, Superconductivity in a layered perovskite without copper, Nature (London) 372, 532 (1994).

[2] Y. Maeno, T. M. Rice, and M. Sigrist, The intriguing superconductivity of strontium ruthenate, Phys. Today 54(1), 42 (2001).

[3] A. P. Mackenzie and Y. Maeno, The superconductivity of $\mathrm{Sr}_{2} \mathrm{RuO}_{4}$ and the physics of spin-triplet pairing, Rev. Mod. Phys. 75, 657 (2003).

[4] C. Kallin and A. J. Berlinsky, Is $\mathrm{Sr}_{2} \mathrm{RuO}_{4}$ a chiral p-wave superconductor?, J. Phys. Condens. Matter 21, 164210 (2009).

[5] C. Kallin, Chiral p-wave order in $\mathrm{Sr}_{2} \mathrm{RuO}_{4}$, Rep. Prog. Phys. 75, 042501 (2012).

[6] Y. Maeno, S. Kittaka, T. Nomura, S. Yonezawa, and K. Ishida, Evaluation of spin-triplet superconductivity in $\mathrm{Sr}_{2} \mathrm{RuO}_{4}$, J. Phys. Soc. Jpn. 81, 011009 (2012).

[7] Y. Liu and Z. Q. Mao, Unconventional superconductivity in $\mathrm{Sr}_{2} \mathrm{RuO}_{4}$, Physica C 514, 339 (2015).

[8] C. Kallin and A. J. Berlinsky, Chiral superconductors, Rep. Prog. Phys. 79, 054502 (2016).

[9] A. P. Mackenzie, T. Scaffidi, C. W. Hicks, and Y. Maeno, Even odder after twenty-three years: The superconducting or- der parameter puzzle of $\mathrm{Sr}_{2} \mathrm{RuO}_{4}$, npj Quantum Mater. 2, 40 (2017).

[10] A. J. Leggett and Y. Liu, Symmetry properties of superconducting order parameter in $\mathrm{Sr}_{2} \mathrm{RuO}_{4}$, J. Supercond. Nov. Magn. 34, 1647 (2021).

[11] G. M. Luke, Y. Fudamoto, K. M. Kojima, M. I. Larkin, J. Merrin, B. Nachumi, Y. J. Uemura, Y. Maeno, Z. Q. Mao, Y. Mori, H. Nakamura, and M. Sigrist, Time-reversal Symmetrybreaking Superconductivity in $\mathrm{Sr}_{2} \mathrm{RuO}_{4}$, Nature (London) 394, 558 (1998).

[12] V. Grinenko, S. Ghosh, R. Sarkar, J. Orain, A. Nikitin, M. Elender, D. Das, Z. Guguchia, F. Brückner, M. E. Barber, J. Park, N. Kikugawa, D. A. Sokolov, J. S. Bobowski, T. Miyoshi, Y. Maeno, A. P. Mackenzie, H. Luetkens, C. W. Hicks, and H. Klauss, Split superconducting and time-reversal symmetrybreaking transitions in $\mathrm{Sr}_{2} \mathrm{RuO}_{4}$ under stress, Nat. Phys. 17, 748 (2021).

[13] J. Xia, Y. Maeno, P. T. Beyersdorf, M. M. Fejer, and A. Kapitulnik, High Resolution Polar Kerr Effect Measurements of $\mathrm{Sr}_{2} \mathrm{RuO}_{4}$ : Evidence for Broken Time-Reversal Symmetry in the Superconducting State, Phys. Rev. Lett. 97, 167002 (2006). 
[14] F. Kidwingira, J. D. Strand, D. J. van Harlingen, and Y. Maeno, Dynamical superconducting order parameter domains in $\mathrm{Sr}_{2} \mathrm{RuO}_{4}$, Science 314, 1267 (2006).

[15] S. Ghosh, A. Shekhter, F. Jerzembeck, N. Kikugawa, D. A. Sokolov, M. Brando, A. P. Mackenzie, C. W. Hicks, and B. J. Ramshaw, Thermodynamic evidence for a two-component superconducting order parameter in $\mathrm{Sr}_{2} \mathrm{RuO}_{4}$, Nat. Phys. 17, 199 (2021).

[16] S. Benhabib, C. Lupien, I. Paul, L. Berges, M. Dion, M. Nardone, A. Zitouni, Z. Q. Mao, Y. Maeno, A. Georges, L. Taillefer, and C. Proust, Ultrasound evidence for a twocomponent superconducting order parameter in $\mathrm{Sr}_{2} \mathrm{RuO}_{4}$, Nat. Phys. 17, 194 (2021).

[17] J-L. Zhang, Y. Li, W. Huang, and F-C. Zhang, Hidden anomalous Hall effect in $\mathrm{Sr}_{2} \mathrm{RuO}_{4}$ with chiral superconductivity dominated by the Ru $d_{x y}$ orbital, Phys. Rev. B 102, 180509(R) (2020).

[18] A. Pustogow, Y. Luo, A. Chronister, Y. Su, D. A. Sokolov, F. Jerzembeck, A. P. Mackenzie, C. W. Hicks, N. Kikugawa, S. Raghu, E. D. Bauer, and S. E. Brown, Constraints on the superconducting order parameter in $\mathrm{Sr}_{2} \mathrm{RuO}_{4}$ from oxygen17 nuclear magnetic resonance, Nature (London) 574, 72 (2019).

[19] K. Ishida, M. Manago, and Y. Maeno, Reduction of the ${ }^{17} \mathrm{O}$ knight shift in the superconducting state and the heat-up effect by NMR pulses on $\mathrm{Sr}_{2} \mathrm{RuO}_{4}$, J. Phys. Soc. Jpn. 89, 034712 (2020).

[20] J. R. Kirtley, C. Kallin, C. W. Hicks, E.-A. Kim, Y. Liu, K. A. Moler, Y. Maeno, and K. D. Nelson, Upper limit on spontaneous supercurrents in $\mathrm{Sr}_{2} \mathrm{RuO}_{4}$, Phys. Rev. B 76, 014526 (2007).

[21] C. W. Hicks, J. R. Kirtley, T. M. Lippman, N. C. Koshnick, M. E. Huber, Y. Maeno, W. M. Yuhasz, M. B. Maple, and K. A. Moler, Limits on superconductivity-related magnetization in $\mathrm{Sr}_{2} \mathrm{RuO}_{4}$ and $\mathrm{PrOs}_{4} \mathrm{Sb}_{12}$ from Scanning SQUID Microscopy, Phys. Rev. B 81, 214501 (2010).

[22] D. Vollhardt and P. Wölfle, The Superfluid Phases of Helium 3 (Dover, New York, 1990).

[23] K. D. Nelson, Z. Q. Mao, Y. Maeno, and Y. Liu, Odd-Parity Superconductivity in $\mathrm{Sr}_{2} \mathrm{RuO}_{4}$, Science 306, 1151 (2004).

[24] J. F. Annett, G. Litak, B. L. Györffy, and K. I. Wysokiński, Spin-orbit coupling and symmetry of the order parameter in strontium ruthenate, Phys. Rev. B 73, 134501 (2006).

[25] T. Scaffidi, J. C. Romers, and S. H. Simon, Pairing symmetry and dominant B band in $\mathrm{Sr}_{2} \mathrm{RuO}_{4}$, Phys. Rev. B 89, 220510(R) (2014).

[26] L. D. Zhang, W. Huang, F. Yang, and H. Yao, Superconducting pairing in $\mathrm{Sr}_{2} \mathrm{RuO}_{4}$ from weak to intermediate coupling, Phys. Rev. B 97, 060510(R) (2018).

[27] A. T. Rømer, D. D. Scherer, I. M. Eremin, P. J. Hirschfeld, and B. M. Andersen, Knight Shift and Leading Superconducting Instability from Spin Fluctuations in $\mathrm{Sr}_{2} \mathrm{RuO}_{4}$, Phys. Rev. Lett. 123, 247001 (2019).

[28] A. T. Rømer, D. D. Scherer, I. M. Eremin, P. J. Hirschfeld, and B. M. Andersen, Fluctuation-driven Superconductivity in $\mathrm{Sr}_{2} \mathrm{RuO}_{4}$ from weak repulsive interactions, Mod. Phys. Lett. B 34, 2040052 (2020).

[29] H. S. Roising, T. Scaffidi, F. Flicker, G. F. Lange, and S. H. Simon, Superconducting order of $\mathrm{Sr}_{2} \mathrm{RuO}_{4}$ from a threedimensional microscopic model, Phys. Rev. Research 1, 033108 (2019).
[30] Z. Wang, X. Wang, and C. Kallin, Spin-orbit coupling and spintriplet pairing symmetry in $\mathrm{Sr}_{2} \mathrm{RuO}_{4}$, Phys. Rev. B 101, 064507 (2020).

[31] N. Read and D. Green, Paired states of fermions in two dimensions with breaking of parity and time-reversal symmetries and the fractional quantum hall effect, Phys. Rev. B 61, 10267 (2000).

[32] E. Taylor and C. Kallin, Intrinsic Hall Effect in a Multiband Chiral Superconductor in the Absence of an External Magnetic Field, Phys. Rev. Lett. 108, 157001 (2012).

[33] K. I. Wysokiński, J. F. Annett, and B. L. Györffy, Intrinsic Optical Dichroism in the Chiral Superconducting State of $\mathrm{Sr}_{2} \mathrm{RuO}_{4}$, Phys. Rev. Lett. 108, 077004 (2012).

[34] Z. Wang, J. Berlinsky, G. Zwicknagl, and C. Kallin, Intrinsic Ac anomalous hall effect of nonsymmorphic chiral superconductors with an application to $\mathrm{UPt}_{3}$, Phys. Rev. B 96, 174511 (2017).

[35] See Supplemental Material at http://link.aps.org/supplemental/ 10.1103/PhysRevResearch.3.L042002 for details of the calculation of $\sigma_{\mathrm{H}}$ and $\theta_{\mathrm{K}}$, and further explanations of the absence of cross-gradient terms in the GL free energy that are relevant to spontaneous current.

[36] M. Matsumoto and M. Sigrist, Quasiparticle states near the surface and the domain wall in a $p_{x} \pm i p_{y}$-wave superconductor, J. Phys. Soc. Jpn. 68, 994 (1999).

[37] A. Furusaki, M. Matsumoto, and M. Sigrist, Spontaneous hall effect in a chiral p-wave superconductor, Phys. Rev. B 64, 054514 (2001).

[38] P. E. C. Ashby and C. Kallin, Suppression of spontaneous supercurrents in a chiral P-wave superconductor, Phys. Rev. B 79, 224509 (2009).

[39] Y. Imai, K. Wakabayashi, and M. Sigrist, Properties of edge states in a spin-triplet two-band superconductor, Phys. Rev. B 85, 174532 (2012).

[40] Y. Imai, K. Wakabayashi, and M. Sigrist, Topological and edge state properties of a three-band model for $\mathrm{Sr}_{2} \mathrm{RuO}_{4}$, Phys. Rev. B 88, 144503 (2013).

[41] S. Lederer, W. Huang, E. Taylor, S. Raghu, and C. Kallin, Suppression of spontaneous currents in $\mathrm{Sr}_{2} \mathrm{RuO}_{4}$ by surface disorder, Phys. Rev. B 90, 134521 (2014).

[42] A. Bouhon and M. Sigrist, Current inversion at the edges of a chiral P-wave superconductor, Phys. Rev. B 90, 220511(R) (2014).

[43] W. Huang, S. Lederer, E. Taylor, and C. Kallin, Nontopological nature of the edge current in a chiral $\mathrm{P}$-wave superconductor, Phys. Rev. B 91, 094507 (2015).

[44] T. Scaffidi and S. H. Simon, Large Chern Number and Edge Currents in $\mathrm{Sr}_{2} \mathrm{RuO}_{4}$, Phys. Rev. Lett. 115, 087003 (2015).

[45] M. Sigrist and K. Ueda, Phenomenological theory of unconventional superconductivity, Rev. Mod. Phys. 63, 239 (1991).

[46] X. Wang, Z. Wang, and C. Kallin, Spontaneous edge current in higher chirality superconductors, Phys. Rev. B 98, 094501 (2018).

[47] S. A. Kivelson, A. C. Yuan, B. J. Ramshaw, and R. Thomale, A proposal for reconciling diverse experiments on the superconducting state in $\mathrm{Sr}_{2} \mathrm{RuO}_{4}$, npj Quantum Mater. 5, 43 (2020).

[48] A. N. Petsch, M. Zhu, M. Enderle, Z. Q. Mao, Y. Maeno, I. I. Mazin, and S. M. Hayden, Reduction of the Spin Susceptibility in the Superconducting State of $\mathrm{Sr}_{2} \mathrm{RuO}_{4}$ Observed by Polarized Neutron Scattering, Phys. Rev. Lett. 125, 217004 (2020). 
[49] A. Chronister, A. Pustogow, N. Kikugawa, D. A. Sokolov, F. Jerzembeck, C. W. Hicks, A. P. Mackenzie, E. D. Bauer, and S. E. Brown, Evidence for even parity unconventional superconductivity in $\mathrm{Sr}_{2} \mathrm{RuO}_{4}$, Proc. Natl. Acad. Sci. (USA) 118, e2025313118 (2021).

[50] W. Huang, Y. Zhou, and H. Yao, Exotic Cooper pairing in Multiorbital Models of $\mathrm{Sr}_{2} \mathrm{RuO}_{4}$, Phys. Rev. B 100, 134506 (2019).

[51] A. Ramires and M. Sigrist, Superconducting order parameter of $\mathrm{Sr}_{2} \mathrm{RuO}_{4}$ : A microscopic perspective, Phys. Rev. B 100, 104501 (2019).

[52] S. O. Kaba and D. Sénéchal, Group-theoretical classification of superconducting states of strontium ruthenate, Phys. Rev. B 100, 214507 (2019).

[53] C. M. Puetter and H.-Y. Kee, Identifying spin-triplet pairing in spin-orbit coupled multi-band superconductors, Europhys. Lett. 98, 27010 (2012).

[54] A. J. Leggett, Spin Susceptibility of a Superfluid Fermi Liquid, Phys. Rev. Lett. 14, 536 (1965).

[55] P. Steffens, Y. Sidis, J. Kulda, Z. Q. Mao, Y. Maeno, I. I. Mazin, and M. Braden, Spin Fluctuations in $\mathrm{Sr}_{2} \mathrm{RuO}_{4}$ from Polarized Neutron Scattering: Implications for Superconductivity, Phys. Rev. Lett. 122, 047004 (2019).

[56] E. Pavarini and I. I. Mazin, First-principles study of spin-orbit effects and NMR in $\mathrm{Sr}_{2} \mathrm{RuO}_{4}$, Phys. Rev. B 74, 035115 (2006).

[57] C. W. Hicks, D. O. Brodsky, E. A. Yelland, et al., Strong increase of $T_{c}$ of $\mathrm{Sr}_{2} \mathrm{RuO}_{4}$ under both tensile and compressive strain, Science 344, 283 (2014).

[58] A. Steppke, L. Zhao, M. E. Barber, T. Scaffidi, et al., Strong peak in $T_{c}$ of $\mathrm{Sr}_{2} \mathrm{RuO}_{4}$ under uniaxial pressure, Science 355, eaaf9398 (2017)

[59] C. A. Watson, A. S. Gibbs, A. P. Mackenzie, C. W. Hicks, and K. A. Moler, Micron-scale measurements of low anisotropic strain response of local $T_{c}$ in $\mathrm{Sr}_{2} \mathrm{RuO}_{4}$, Phys. Rev. B 98, 094521 (2018).

[60] S. Yonezawa, T. Kajikawa, and Y. Maeno, Specific-heat evidence of the first-order superconducting transition in $\mathrm{Sr}_{2} \mathrm{RuO}_{4}$, J. Phys. Soc. Jpn. 83, 083706 (2014).

[61] Y.-S. Li, N. Kikugawa, D. A. Sokolov, F. Jerzembeck, A. S. Gibbs, Y. Maeno, C. W. Hicks, M. Nicklas, and A. P. Mackenzie, High-sensitivity Heat-capacity Measurements on $\mathrm{Sr}_{2} \mathrm{RuO}_{4}$ under Uniaxial Pressure, Proc. Natl. Acad. Sci. (USA) 118, e2020492118 (2021).

[62] T. Scaffidi, Degeneracy between even- and odd-parity superconductivity in the quasi-1D hubbard model and implications for $\mathrm{Sr}_{2} \mathrm{RuO}_{4}$, arXiv:2007.13769.

[63] R. Gupta, T. Saunderson, S. Shallcross, M. Gradhand, J. Quintanilla, and J. Annett, Superconducting subphase and substantial knight shift in $\mathrm{Sr}_{2} \mathrm{RuO}_{4}$, Phys. Rev. B 102, 235203 (2020).

[64] K. Deguchi, M. A. Tanatar, Z. Q. Mao, T. Ishiguro, and Y. Maeno, Superconducting double transition and the upper critical field limit of $\mathrm{Sr}_{2} \mathrm{RuO}_{4}$ in parallel magnetic fields, J. Phys. Jpn. Soc. 71, 2839 (2002).

[65] S. Yonezawa, T. Kajikawa, and Y. Maeno, First-Order Superconducting Transition of $\mathrm{Sr}_{2} \mathrm{RuO}_{4}$, Phys. Rev. Lett. 110, 077003 (2013).
[66] K. Deguchi, Z. Q. Mao, H. Yaguchi, and Y. Maeno, Gap Structure of the Spin-Triplet Superconductor $\mathrm{Sr}_{2} \mathrm{RuO}_{4}$ Determined from the Field-Orientation Dependence of the Specific Heat, Phys. Rev. Lett. 92, 047002 (2004).

[67] S. Nishizaki, Y. Maeno, and Z. Q. Mao, Changes in the superconducting state of $\mathrm{Sr}_{2} \mathrm{RuO}_{4}$ under magnetic fields probed by specific heat, J. Phys. Soc. Jpn. 69, 572 (2000).

[68] I. A. Firmo, S. Lederer, C. Lupien, A. P. Mackenzie, J. C. Davis, and S. A. Kivelson, Evidence from tunneling spectroscopy for a quasi-one-dimensional origin of superconductivity in $\mathrm{Sr}_{2} \mathrm{RuO}_{4}$, Phys. Rev. B 88, 134521 (2013).

[69] E. Hassinger, P. Bourgeois-Hope, H. Taniguchi, S. Rene de Cotret, G. Grissonnanche, M. S. Anwar, Y. Maeno, N. DoironLeyraud, and Louis Taillefer, Vertical Line Nodes in the Superconducting Gap Structure of $\mathrm{Sr}_{2} \mathrm{RuO}_{4}$, Phys. Rev. X 7 , 011032 (2017).

[70] J. F. Dodaro, Z. Wang, and C. Kallin, Effects of deep superconducting gap minima and disorder on residual thermal transport in $\mathrm{Sr}_{2} \mathrm{RuO}_{4}$, Phys. Rev. B 98, 214520 (2018).

[71] D. F. Agterberg, T. M. Rice, and M. Sigrist, Orbital Dependent Superconductivity in $\mathrm{Sr}_{2} \mathrm{RuO}_{4}$, Phys. Rev. Lett. 78, 3374 (1997).

[72] M. E. Zhitomirsky and T. M. Rice, Interband Proximity Effect and Nodes of Superconducting Gap in $\mathrm{Sr}_{2} \mathrm{RuO}_{4}$, Phys. Rev. Lett. 87, 057001 (2001).

[73] A. Ramires and M. Sigrist, Identifying detrimental effects for multiorbital superconductivity: Application to $\mathrm{Sr}_{2} \mathrm{RuO}_{4}$, Phys. Rev. B 94, 104501 (2016).

[74] W.-S. Wang, C.-C. Zhang, F.-C. Zhang, and Q.-H. Wang, Theory of Chiral P-Wave Superconductivity with Near Nodes for $\mathrm{Sr}_{2} \mathrm{RuO}_{4}$, Phys. Rev. Lett. 122, 027002 (2019).

[75] O. Gingras, R. Nourafkan, A. M. S. Tremblay, and M. Còté, Superconducting Symmetries of $\mathrm{Sr}_{2} \mathrm{RuO}_{4}$ from First-Principles Electronic Structure, Phys. Rev. Lett. 123, 217005 (2019).

[76] Y. Li and W. Huang, Possible 'symmetry-imposed' near-nodal two-dimensional p-wave pairing in $\mathrm{Sr}_{2} \mathrm{RuO}_{4}$, arXiv:1909.03141.

[77] H. G. Suh, H. Menke, P. M. R. Brydon, C. Timm, A. Ramires, and D. F. Agterberg, Stabilizing even-parity chiral superconductivity in $\mathrm{Sr}_{2} \mathrm{RuO}_{4}$, Phys. Rev. Research 2, 032023(R) (2020).

[78] W. Chen and J. An, interorbital p- and d-wave pairings between $d_{x z} / d_{y z}$ and $d_{x y}$ orbitals in $\mathrm{Sr}_{2} \mathrm{RuO}_{4}$, Phys. Rev. B 102, 094501 (2020).

[79] A. W. Lindquist and H-Y. Kee, Distinct reduction of knight shift in superconducting state of $\mathrm{Sr}_{2} \mathrm{RuO}_{4}$ under uniaxial strain, Phys. Rev. Research 2, 032055(R) (2020).

[80] A. Sumiyama, D. Kawakatsu, J. Gouchi, A. Yamaguchi, G. Motoyama, Y. Hirose, R. Settai, and Y. Ōnuki, Search for spontaneous magnetization of superconductors with broken time-reversal symmetry, JPS Conf. Proc. 3, 015017 (2014).

[81] R. Willa, M. Hecker, R. M. Fernandes, and J. Schmalian, Inhomogeneous time-reversal symmetry breaking in $\mathrm{Sr}_{2} \mathrm{RuO}_{4}$, Phys. Rev. B 104, 024511 (2021).

[82] L-H. Hu, X. Wang, and T. Shang, Spontaneous magnetization in unitary superconductors with time reversal symmetry breaking, Phys. Rev. B 104, 054520 (2021). 